BNL-114504-2014-IR

CBETA/000

December 2014

\title{
A white paper: \\ The Cornell-BNL FFAG-ERL Test Accelerator
}

\author{
I. Ben-Zvi, S. Berg, M. Blaskiewicz, S. Brooks, K. Brown, \\ W. Fischer, Y. Hao, W. Meng, F. Meot, M. Minty, S. Peggs, \\ V. Ptitsin, T. Roser, P. Thieberger, D. Trbojevic, N. Tsoupas, \\ I. Bazarov, J. Dobbins, B. Dunham, G. Hoffstaetter, \\ C. Mayes, R. Patterson, D. Sagan
}

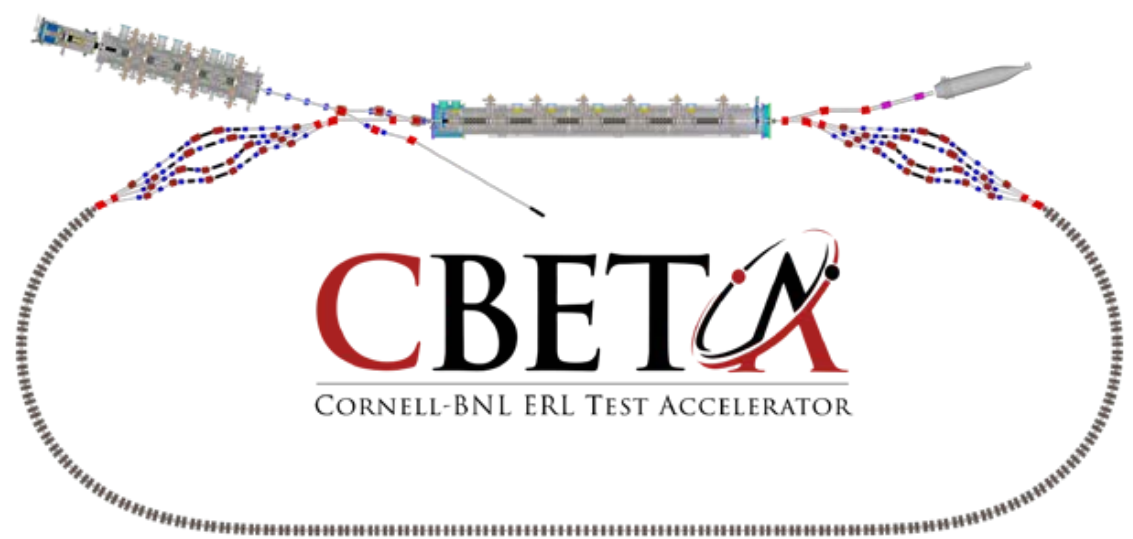

Collider-Accelerator Department, Brookhaven National Laboratory, Upton NY 11973 U.S. Department of Energy

Office of Science, Office of Nuclear Physics

Cornell Laboratory for Accelerator-Based Sciences and Education, Ithaca, NY 14850

Funded by NYSERDA contract 102192

Notice: This document has been authorized by employees of Brookhaven Science Associates, LLC under Contract No. DE-SC0012704 with the U.S. Department of Energy. The United States Government retains a non-exclusive, paid-up, irrevocable, world-wide license to publishor reproduce the published form of this document, or allow others to do so, for United States Government purposes. 


\section{DISCLAIMER}

This report was prepared as an account of work sponsored by an agency of the United States Government. Neither the United States Government nor any agency thereof, nor any of their employees, nor any of their contractors, subcontractors, or their employees, makes any warranty, express or implied, or assumes any legal liability or responsibility for the accuracy, completeness, or any third party's use or the results of such use of any information, apparatus, product, or process disclosed, or represents that its use would not infringe privately owned rights. Reference herein to any specific commercial product, process, or service by trade name, trademark, manufacturer, or otherwise, does not necessarily constitute or imply its endorsement, recommendation, or favoring by the United States Government or any agency thereof or its contractors or subcontractors. The views and opinions of authors expressed herein do not necessarily state or reflect those of the United States Government or any agency thereof. 


\section{A white paper: The Cornell-BNL FFAG-ERL Test Accelerator}

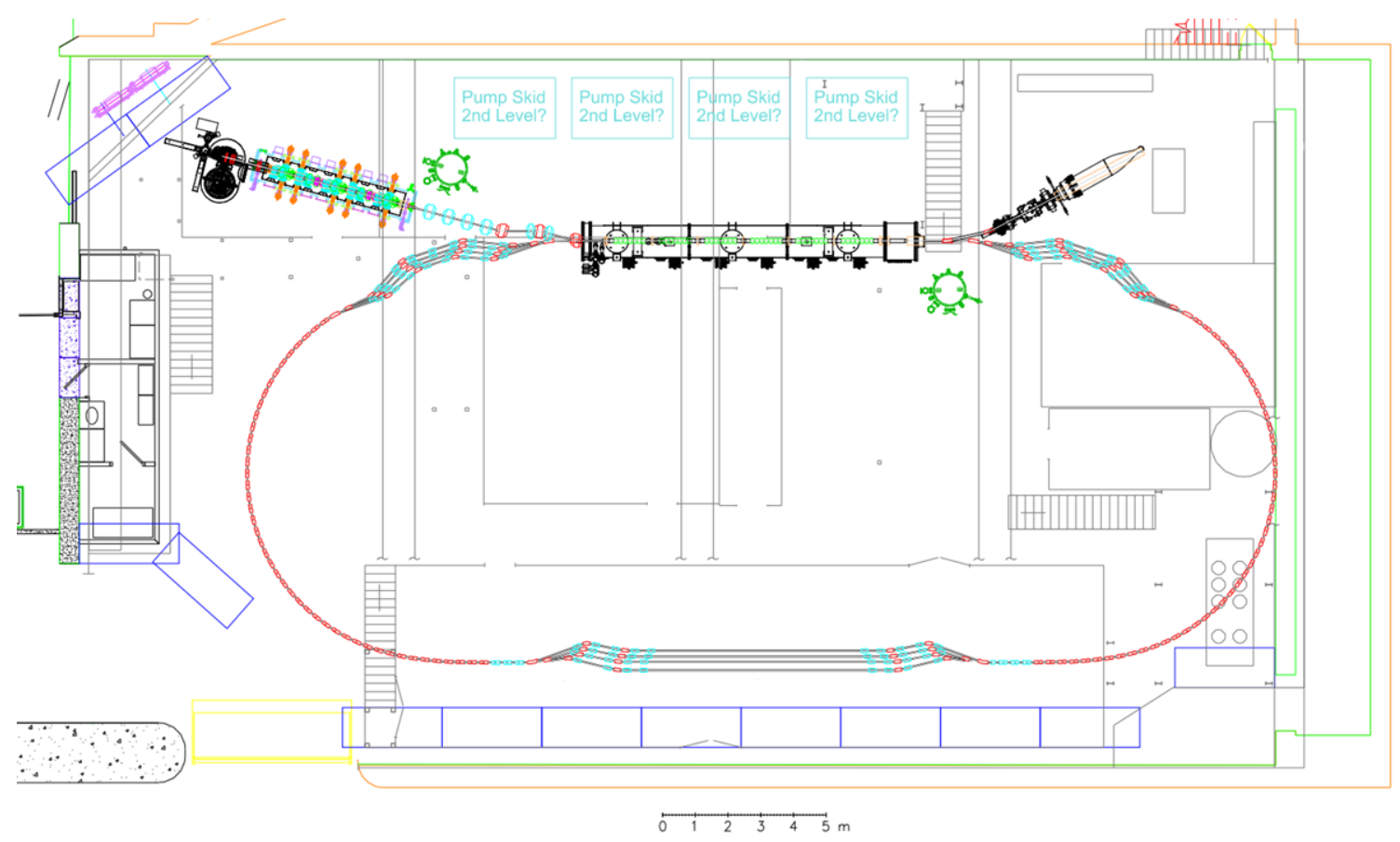

BNL: Ilan Ben-Zvi, Scott Berg, Michael Blaskiewicz, Stephen Brooks, Kevin Brown, Wolfram Fischer, Yue Hao, Wuzheng Meng, François Méot, Michiko Minty, Stephen Peggs, Vadim Ptitsin, Thomas Roser, Peter Thieberger, Dejan Trbojevic, Nick Tsoupas.

Cornell: Ivan Bazarov, John Dobbins, Bruce Dunham, Georg Hoffstaetter, Christopher Mayes, Ritchie Patterson, David Sagan.

December 16, 2014 


\section{Executive summary}

The Cornell-BNL FFAG-ERL Test Accelerator (C $\beta$ ) will be a unique resource to carry out accelerator science and enable exciting research in nuclear physics, materials science and industrial applications.

$\mathrm{C} \beta$ will comprise the first ever Energy Recovery Linac (ERL) based on a Fixed Field Alternating Gradient (FFAG) lattice. In particular, we plan to use a Non Scaling FFAG (NS-FFAG) lattice that is very compact and thus space- and cost- effective, enabling multiple passes of the electron beam in a single recirculation beam line, using the superconducting RF (SRF) linac multiple times. The FFAG-ERL moves the cost optimized linac and recirculation lattice to a dramatically better optimum.

The prime accelerator science motivation for $\mathrm{C} \beta$ is proving that the FFAG-ERL concept works. This is an important milestone for the BNL plans to build a major Nuclear Physics facility, eRHIC, based on producing $21 \mathrm{GeV}$ electron beams to collide with the RHIC ion beams [1]. A consequence of the $C \beta$ work would be the availability of significantly better, cost-effective, compact CW high-brightness electron beams for a plethora of scientific investigations and applications, such as Xray sources, dark-matter and dark-energy searches, industrial high-power FreeElectron Laser (FEL) applications, and much more.

$\mathrm{C} \beta$ brings together the resources and expertise of a large DOE National Laboratory, $\mathrm{BNL}$, and a leading research university, Cornell. $\mathrm{C} \beta$ will be built in an existing building at Cornell, for the most part using components that have been developed under previous R\&D programs, including a fully commissioned world-leading photoemission electron injector, a large SRF accelerator module, and a high-power beam stop. The only elements that require design and construction from scratch is the FFAG magnet transport lattice.

This white paper describes a project that promises to propel high-power, highbrightness electron beam science and applications to an exciting new level. The collaborative effort between Brookhaven and Cornell will be a model for future projects between universities and national lab, taking advantage of the expertise and resources of both to investigate new topics in a timely and cost-effective manner. 


\section{Introduction}

\section{Primary motivations}

BNL is planning to transform RHIC to eRHIC by installing a new electron accelerator in the existing tunnel, providing polarized electrons with energies from $5 \mathrm{GeV}$ to 21 $\mathrm{GeV}$ for collisions with polarized protons, polarized ${ }^{3} \mathrm{He}$ ions, or with ions from deuterons to Uranium. Significant simplification and cost reduction is possible by configuring eRHIC with non-scaling, Fixed-Field Alternating Gradient (NS-FFAG) optics in combination with an ERL. Two NS-FFAG beamline arcs placed on top of each other allow multiple passes through a single superconducting linac. Such magnets and optical techniques will be prototyped in the Cornell-BNL FFAG-ERL Test Accelerator $(\mathrm{C} \beta)$, along with other innovative accelerator technologies, commissioning eRHIC instrumentation and confirming the theories for multi-pass recirculative beam breakup.

The high beam power and high brightness provided by $\mathrm{C} \beta$ will also enable exciting and important physics experiments including dark matter and dark energy searches [2], Q-weak tests at lower energies [3], proton charge radius measurements, and an array of polarized-electron enabled nuclear physics experiments. High brightness, narrow line width gamma rays can be generated by Compton scattering [4] using the ERL beam, and can be used for nuclear resonance fluorescence, the detection of special nuclear materials, and an array of astrophysical measurements. The energy and current range of $\mathrm{C} \beta$ will also be ideal for studying high power FEL physics for materials research and for industrial uses.

\section{The LOE experimental hall at Cornell}

Figure 1 shows the floor plan layout of a preliminary C $\beta$ lattice, in the LOE experimental hall in Wilson laboratory at Cornell, while Table 1 lists the primary accelerator parameters. Figure 1 illustrates that there is sufficient space for all of the required hardware. At the bottom and at the right of the accelerator are external walls. The upper wall separates the area from the Cornell Electron Storage Ring (CESR), while on the left side are X-ray stations of the Cornell High Energy Synchrotron Source (CHESS).

The most expensive $\mathrm{C} \beta$ components - the injector and the linac cryomodule - are already built and are available for use at Cornell. The LOE location is already served by significant accelerator infrastructure and other resources, including a liquid helium plant. $\mathrm{C} \beta$ is an important part of future plans at Cornell for accelerator research, nuclear physics research, materials studies, and ERL studies. 


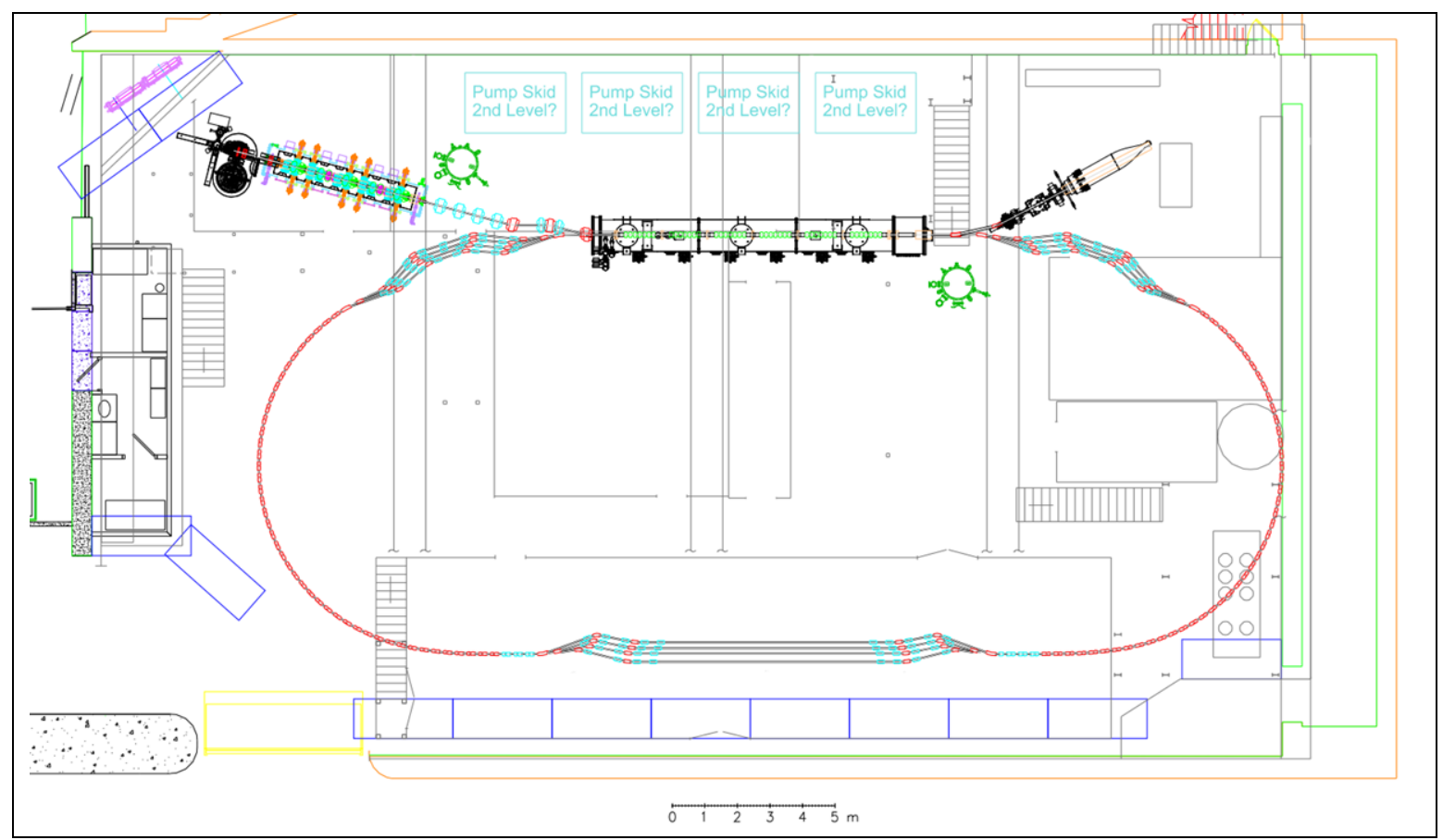

Figure 1 Possible floor plan of the Cornell-BNL FFAG-ERL Test Accelerator in the LOE experimental hall in Wilson laboratory.

Table 1 Primary parameters of the Cornell-BNL FFAG-ERL Test Accelerator.

\begin{tabular}{|l|c|l|}
\hline \multicolumn{1}{|c|}{ Parameter } & Unit & \multicolumn{1}{c|}{ Value } \\
\hline Linac energy gain & $\mathrm{MeV}$ & 70 \\
Injection energy & $\mathrm{MeV}$ & 6 \\
Linac passes & & 8 total: 4 accelerating + 4 decelerating \\
7 energy sequence & $\mathrm{MeV}$ & $76,146,216,286,216,146,76$ \\
RF frequency & $\mathrm{MHz}$ & 1300 \\
RF period & $\mathrm{ns}$ & 0.7692 \\
Circumference harmonic & & 279 \\
Accelerator circumference & $\mathrm{m}$ & 64.34 \\
Revolution period & $\mu \mathrm{s}$ & 0.2146 \\
Electron gun current & $\mathrm{mA}$ & 100 \\
Normalised RMS emittance & $\mu \mathrm{m}$ & 2 (at $1 \mathrm{nC})$ \\
Typical arc beta functions & $\mathrm{m}$ & 0.5 (ranges from 0.05 to 2.5) \\
Typical RMS beam size & $\mu \mathrm{m}$ & 50 (ranges from 20 to 80) \\
Bunch length & $\mathrm{ps}$ & 3 \\
Single bunch charge & & \\
$\quad$ Initial commissioning & $\mathrm{nC}$ & $\sim 0.1$ \\
$\quad$ Physics operation (CW) & $\mathrm{nC}$ & 0.1 to 1.0 \\
Dedicated experiments & $\mathrm{nC}$ & $<5$ \\
\hline
\end{tabular}




\section{NS-FFAG optics in an ERL}

A first NS-FFAG proof-of-principle Electron Model for Multiple Applications (EMMA) was built and operated at Daresbury Laboratory in 2012 [5]. The broader concept of an FFAG was revived recently, but is not new, originally developed by three independent groups in the 1950's $[6,7,8]$. However, FFAG optics have not yet been used in combination with an ERL, a combination that is at the heart of the eRHIC design, and which is a core motivation for $\mathrm{C} \beta$ accelerator physics prototyping. In eRHIC the advantages of NS-FFAG optics are evident: many passes with a shorter superconducting linac and only two electron beamlines in each RHIC arc sector. The $\mathrm{C} \beta$ implementation will enable these prototype magnet and optical technologies to be studied. Advanced understanding of NS-FFAG beam dynamics will also spin-off into related applications, such as hadron therapy, gantry optics, multi-pass neutron generators, and Accelerator Driven Subcritical Reactor (ADSR) systems.

The NS-FFAG concept was originally developed as a solution for fast acceleration of short lifetime muons. Small dispersion functions lead to magnet apertures that are much smaller than with scaling FFAG optics [9]. General advantages include:

1) Energy range factors of 4 or 5 with constant, possibly permanent magnets.

2) Small physical aperture, small magnet size, and small orbit offsets.

3) Linear magnetic fields often enable the use of displaced quadrupoles.

4) Large dynamic apertures, with no non-linear magnets or fields.

5) Extreme focusing - very small Twiss and dispersion functions.

General disadvantages of NS-FFAG optics are:

1) Tune and chromaticity variation with energy.

2) Time-of-flight depends parabolically on beam energy deviation from the central reference energy.

\section{Schedule}

Installation and early prototyping will be performed in several stages over 2 to 3 years, with incremental commissioning occurring as the hardware becomes available. The major schedule stages - with a critical path through magnet delivery - are:

1) Clear out the experimental hall (3 months, year 1)

2) Install the injector and linac cryomodule (3 months, year 1)

3) Install cryogenics and RF power (2 months, year 1)

4) Commission the injector and cryomodule (3-6 months, year 1)

5) Commission single-turn FFAG-ERL (6 months, year 2)

6) Install FFAG magnets and arcs (4 months, year 2)

7) Commission FFAG return loops (year 3)

8) FFAG experiments (year 3)

The injector has already been tested, but needs to be relocated to a new area, recommissioned, and tested - a process of 2 to 3 months. The main linac cryomodule 
will not have undergone any testing before its installation. The first commissioning step is to cool down the entire system to $2 \mathrm{~K}$, taking from one week to one month. Once cold, each cavity will be tested individually using a $5 \mathrm{~kW}$ solid state amplifier. Multi-cavity tests will be performed when more amplifiers are available. Cavity Q's will be measured, and the operation of the tuners, couplers and HOM's will be checked. Testing will take 3 to 6 months.

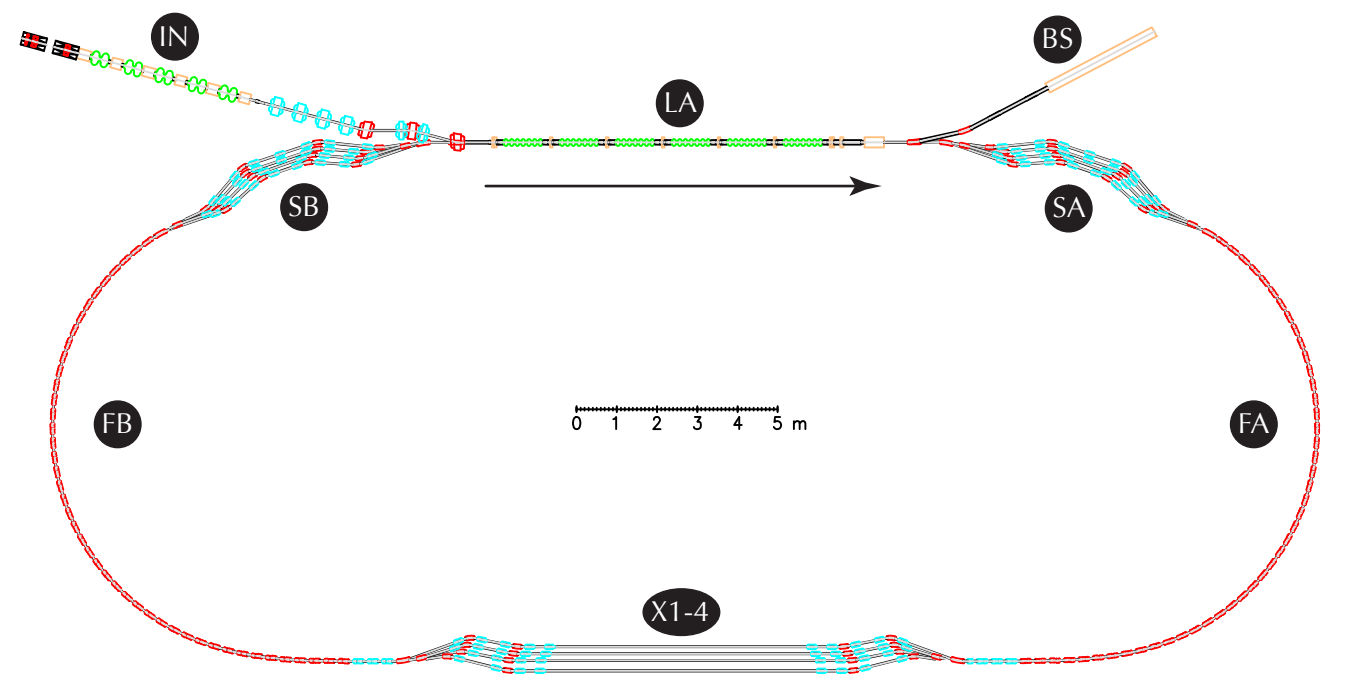

Figure 2 Major optical components. IN - Injector. LA - linac. SA, SB - splittercombiner sections. FA, FB - FFAG arcs. X1-4 - experimental and diagnostic beamline sections. BS - beam stop. 


\section{Optics \& beam dynamics}

Figure 2 shows the major optical components. Starting from the injector (IN), the beam is merged into the linac (LA) at $6 \mathrm{MeV}$, and accelerated in the first pass by $70 \mathrm{MeV}$. This lowest energy beam is separated from beams at other energies when entering the first splitter-combiner section (SA). It merges back with the other beams to travel on the $76 \mathrm{MeV}$ stable orbit in the FFAG arc (FA), before being separated again in the experimental/diagnostic beamline section (X1-4), merging into FFAG arc (FB), followed by a second splitter-combiner section (SB) for matching into another accelerating pass in the linac. The process continues with 4 arc energies of $146 \mathrm{MeV}, 216 \mathrm{MeV}$ and $286 \mathrm{MeV}$, for a total of four accelerating passes. The path length on the fourth pass is chosen so that re-entry into the linac is on the decelerating phase, and so that energy is recovered in the remaining four passes through the linac. Finally the spent $6 \mathrm{MeV}$ beam is directed to the beam stop (BS), which also includes 6D beam diagnostics.

The somewhat complicated splitter-combiner sections are necessary for orbit correction and to match the relatively large transverse beams in the linac to the relatively small beams in the FFAG arcs. They also tune the net path lengths and momentum compaction factors to ensure efficient energy recovery. The experimental area offers space for further optics tuning and diagnostics. The arcs of the racetrack are made of doublet NS-FFAG cells, with a focusing quadrupole QF that is radially displaced by $5.4 \mathrm{~mm}$, and a defocusing magnet BD that could be a displaced quadrupole, or a combined function magnet with only mid-plane symmetry.

Figure 3 shows the radial orbit offsets for the 4 arc energies, and the small values of the matched cell betatron and dispersion functions resulting from the very strong focusing, while Figure 4 shows cross sections of the BD and QF magnets. Table 2 lists the tune-per-cell values and the path length differences for the 4 arc energies, while Table 3 shows the parameters of the arc unit cell.
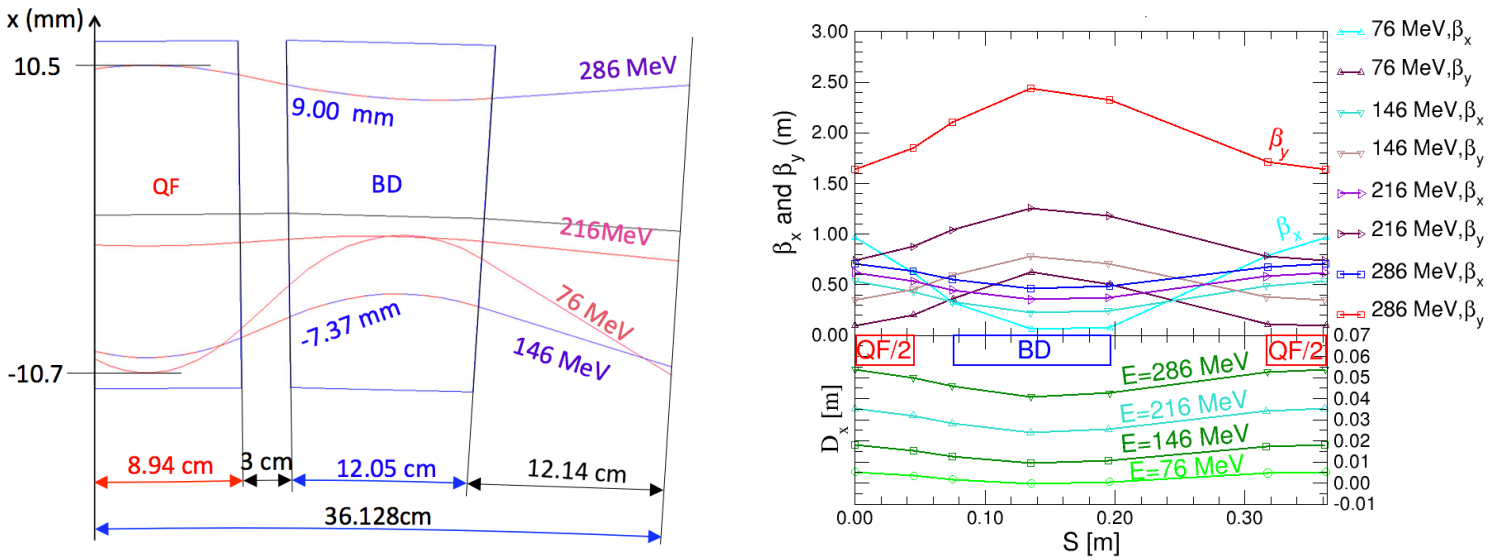

Figure 3 Left: Radial offsets of the design orbits at the 4 arc energies. Right: Matched betatron and dispersion values through the arc cell. 

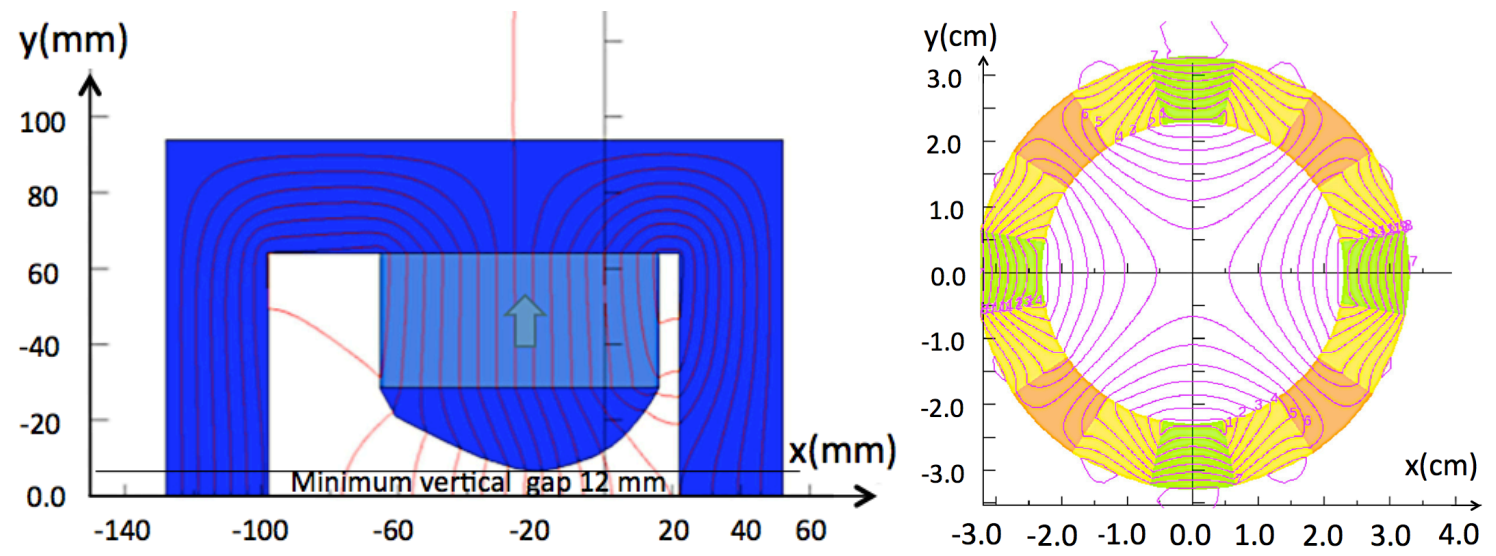

Figure 4 Left: The BD defocusing iron-dominated combined function H-type magnet, with iron in dark blue and permanent magnet material in light blue. Right: The QF focusing Halbach permanent magnet quadrupole, with an overall diameter of about $70 \mathrm{~mm}$. There is ample room for vacuum pipe placement, despite orbit offsets in the range from $-6 \mathrm{~mm}$ to $+16 \mathrm{~mm}$. All systematic multipole errors below 12-pole are zero, thanks to the 4 -fold symmetry.

Table 2 NS-FFAG arc cell behavior. The path length through two FFAG arcs depends parabolically on the deviation of the arc energy from a reference value, because design orbits are radially offset by differing amounts from the arc magnet centers.

\begin{tabular}{|c|cc|c|}
\hline Arc energy & \multicolumn{2}{|c|}{ Tune per cell } & Path length difference \\
{$[\mathrm{MeV}]$} & {$[\mathrm{H}]$} & {$[\mathrm{V}]$} & {$[\mathrm{mm}]$} \\
\hline 76 & 0.406 & 0.282 & 11.6 \\
146 & 0.180 & 0.112 & -81.3 \\
216 & 0.126 & 0.059 & -71.1 \\
286 & 0.100 & 0.033 & 0 \\
\hline
\end{tabular}

Table 3 Lengths and angles of elements in the arc cell, defining a reference curve made of circular arcs and straight lines.

\begin{tabular}{|c|c|c|c|c|}
\hline Element & $\begin{array}{c}\text { Length } \\
{[\mathrm{mm}]}\end{array}$ & $\begin{array}{c}\text { Bend angle } \\
{[\mathrm{mrad}]}\end{array}$ & $\begin{array}{c}\text { Dipole field } \\
{[\mathrm{T}]}\end{array}$ & $\begin{array}{c}\text { Gradient } \\
{[\mathrm{T} / \mathrm{m}]}\end{array}$ \\
\hline QF & 89.4 & 24.219 & 0.21 & -39.1 \\
Drift 1 & 30.0 & & & \\
BD & 120.5 & 87.051 & -0.56 & 25.42 \\
Drift 2 & 121.4 & & & \\
Total & 361.3 & $-2 \pi / 100$ & & \\
\hline
\end{tabular}




\section{Beam breakup}

The beam current in a conventional linear accelerator is typically limited by the electrical power available for acceleration. This limit is lifted in ERLs because the beam energy is fed back into the accelerating fields, but a new limit to the current is created by the beam-breakup instability (BBU). Higher Order Modes (HOMs) excited by the beam passing - more than once - through the SRF cavities give rise to the recirculative beam-breakup instability, when the current is too large, and/or when there are too many passes. This leads to large beam-trajectory oscillations and beam loss. A bunch traversing a cavity with a small dipole-HOM field receives a transverse kick. When this bunch returns to the same cavity it will therefore excite dipole-HOMs, thanks to the transverse offset caused by the initial transverse kick. If it excites the dipole-HOM in phase with the prior HOM field, then the transverse kick will increase and can eventually cause the beam to be lost.

A rigorous theory shows that BBU can become very restrictive in multi-turn ERLs, with a threshold current that decreases with increasing $\mathrm{N}$ as $1 / \mathrm{N}^{*}(2 \mathrm{~N}-1)$, where $\mathrm{N}$ is the total number of accelerating passes [10]. Note that $\mathrm{N}$ can be large - it is 16 in a recent eRHIC design. Comparisons to theory were excellent in the experimental investigations of BBU at the JLAB FEL-ERL - including horizontal-vertical coupling, but only with one pass [11]. Multi-turn BBU has been studied at CEBAF, although not in an ERL [12]. The $C \beta$ accelerator will be the first to experimentally investigate multi-turn BBU in an ERL, a vital activity for eRHIC. Detailed simulations, using the codes developed at Cornell University for the HOM parameters of the designed ERL linac cryomodule [13] show that the BBU threshold for the proposed ERL test is between 40 and $350 \mathrm{~mA}$ depending on the betatron phase advance of the recirculating arcs. Thus, the simulated threshold is sufficiently high for the proposed ERL test loop with $\mathrm{N}=4$. 


\section{Sub-systems}

\section{Injector}

The photoemission electron injector shown in Figure 5 is fully operational, and requires no further development. It has achieved the world-record current of 75 $\mathrm{mA}[14,15,16]$, and record low beam emittances for any CW photoinjector [17], with an equivalent brightness that outperforms the best existing storage rings by a substantial factor, if the injected beam were accelerated to a similar energy. Cornell has established a world-leading effort in photoinjector source development, in the underlying beam theory and simulations, with expertise in guns, photocathodes, and lasers. The strength of the injector group is in combining various cathode advances and innovations with the world's brightest photoinjector. This ability to both formulate the frontier questions for high brightness source development, and also to implement the solutions, makes breakthroughs in accelerator science possible.
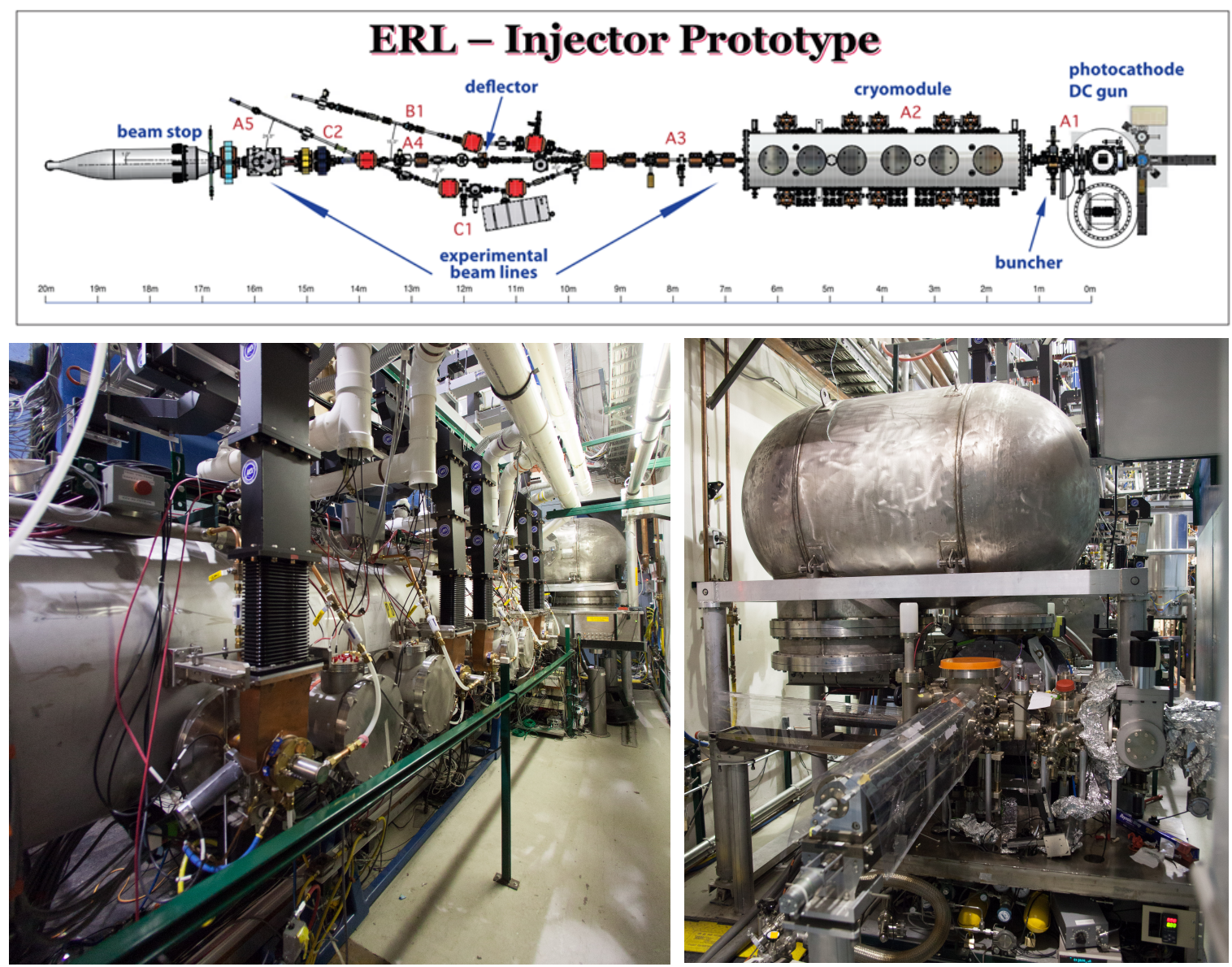

Figure 5 The photoemission injector currently operating at Cornell accelerates the beam to $6 \mathrm{MeV}$. The photographs show (from right to left) the high voltage DC gun, an emittance compensation section, the RF buncher, and the cryomodule. Accelerated beam is then directed into a beamline or into the beam stop. 


\section{SRF cryomodule}

Figure 6 shows the full-scale prototype cryomodule that was developed and constructed as part of the ERL-based X-ray light source project that was proposed at Cornell. It consists of six 7-cell $1300 \mathrm{MHz}$ SRF cavities, including HOM absorbers and RF power couplers. The cavity geometry is carefully designed to maintain high $\mathrm{Q}$ while maximizing the beam breakup threshold current [18]. Cryomodule construction is complete, and the device is available for use in this project. The cryogenics necessary to cool the linac and injector cryomodules are available from the Wilson Lab cryoplant. Additional hardware will be required to transfer the cryogens from the cryoplant to the cryomodules, including heat exchanger cans, valve boxes and $2 \mathrm{~K}$ pump skids.

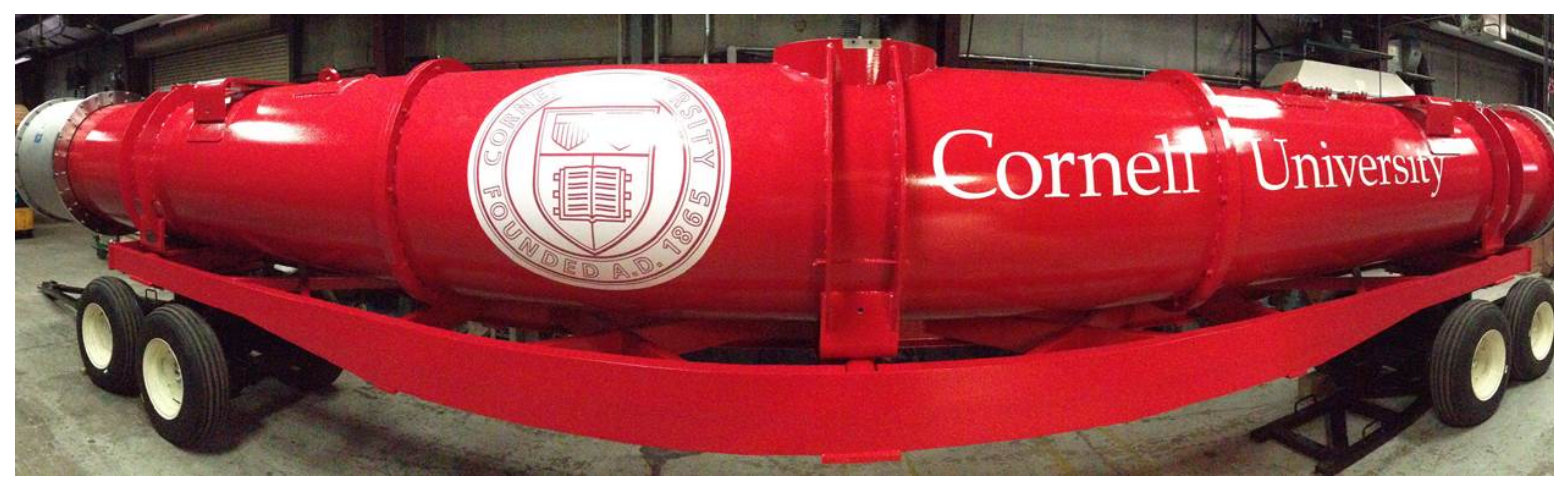

Figure 6 The full-scale prototype $1300 \mathrm{MHz}$ cryomodule that is available for $\mathrm{C} \beta$ use.

\section{RF Power}

The injector delivers up to $500 \mathrm{~kW}$ of RF power to the beam at $1300 \mathrm{MHz}$. The buncher cavity uses a $16 \mathrm{~kW}$ IOT tube, which has adequate overhead for all modes of operation. The injector cryomodule is powered through ten $50 \mathrm{~kW}$ input couplers, using five $130 \mathrm{~kW}$ CW klystrons. The power from each klystron is split to feed two input couplers attached to one individual 2-cell SRF cavity. An additional klystron is available as a backup, or to power a deflection cavity for bunch length measurements. The main linac cryomodule will be powered by 6 individual solidstate RF amplifiers with $5 \mathrm{~kW}$ average power per amplifier. Each cavity has one input coupler. One amplifier is currently available for testing purposes, so an additional 5 amplifiers are needed for this project.

\section{Beam instrumentation and bunch patterns}

Beam instrumentation will support three operational modes:

1) Initial commissioning: one bunch with about $0.1 \mathrm{nC} / \mathrm{bunch}$.

2) Physics operation: CW-like beams, for example with one accelerating (and one decelerating) low charge bunch in every RF period.

3) Dedicated experiment: eRHIC-like trains, typically with bunches spaced by more than 2 ns ( 3 RF buckets), and no more than $5 \mathrm{nC}$ per bunch. 
Figure 7 illustrates bunch pattern behavior in Physics operation mode, observing bunches as they pass a reference point at the end of the linac. Since each bunch is accelerated four times and decelerated four times, the maximum injection rate (that avoids overlapping bunches) is one quarter of the RF frequency. Overlap avoidance also requires the revolution harmonic, 279 , to be an odd number. The maximum energy turn is shorter (or longer) than the other turns by half a wavelength, returning each bunch with the correct phase for deceleration.

The instrumentation design and commissioning strategy for CW, single-pass ERL operation is documented in [19]. With multi-pass ERL operation, a time-isolated "diagnostic" bunch needs to be monitored. Bunch properties - including beam intensity and loss, position, energy, phase, transverse and longitudinal beam sizes will be measured at different energies in the splitter-combiners, experimental straights, and in the beam stop. Optical function measurements enable beam emittance determinations. The broad range of beam parameters in the different operating modes requires careful study to determine if different hardware and/or signal processing electronics will be required.

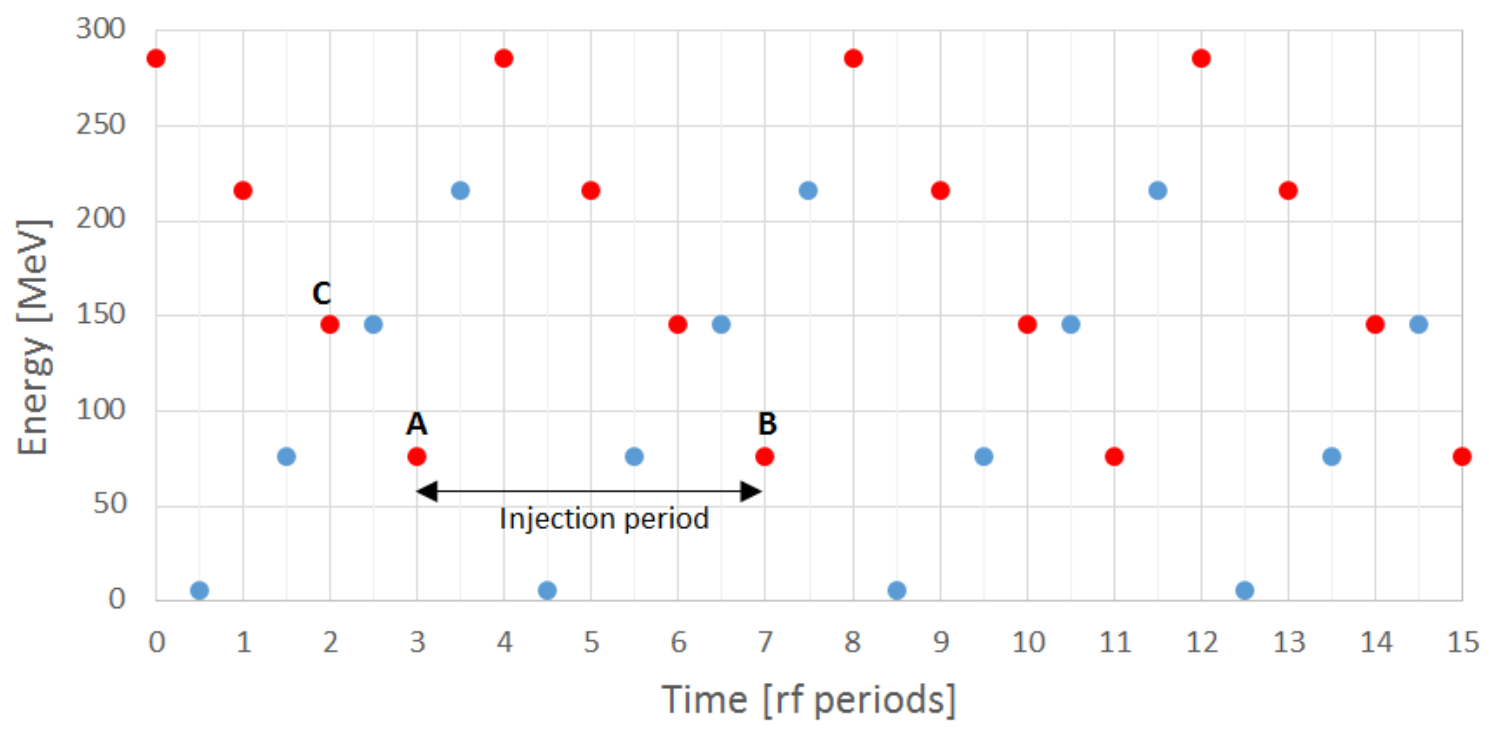

Figure 7 Bunches passing a reference point at the end of the linac, in Physics operation mode with one accelerating bunch (and one decelerating bunch) in every $\mathrm{RF}$ period. Red and blue dots represent accelerating and decelerating bunches, respectively. Bunch " $\mathrm{B}$ ", injected 4 periods after bunch " $\mathrm{A}$ ", also has an energy of 76 $\mathrm{MeV}$. Bunch "C" was injected 280 periods before bunch " $\mathrm{A}$ ", and so has an energy of $146 \mathrm{MeV}$ because it has passed through the linac twice. 
Instrumentation prototyping goals motivated by eRHIC include developing and demonstrating:

1) A high time resolution BPM design. (BNL R\&D is ongoing in FY15/16.)

2) Ion-clearing mechanisms to eliminate conventional ion trapping and fast ion instabilities.

3) High-bandwidth diagnostics for resolving beam sizes in the FFAG cells.

\section{Control system}

The accelerator will use EPICS [20] in order to minimize development time, ensure scalability, and avoid performance limitations. This choice enables the use of hardware and software already developed at Cornell and at BNL. It also provides access to well-defined interfaces at both the server and client levels that are used at many accelerator facilities, since EPICS has a large user base in the accelerator community. EPICS also has well defined developer interfaces and infrastructure allowing rapid development for new systems.

The control system enables supervisory control, automation, and operational analysis, with a scope that extends from the equipment interface to accelerator operators, and to experimenters and technical staff. It includes global systems such as timing, deterministic data communication, network communication, control room operations, automation and optimization, in addition to the computers and software required to implement and integrate all subsystems. The topmost layer of the client-server architecture provides access for non-real time activities, for example high level physics modeling using live or stored data. Accelerator operation and monitoring take place in a middle layer. A dedicated equipment layer, lower down, interfaces to specific equipment through point-to-point protocols. Other low level layers synchronize sub-systems (such as vacuum controls and personnel protection systems) with the accelerator at lower rates. 


\section{Conclusion}

We have described a proposed collaborative effort between Brookhaven National Laboratory and Cornell University to design, build, and test a new type of energy recovery linac.

The FFAG-ERL has the potential to deliver excellent beam quality at a cost that is reasonable, compared to the current state-of-the-art, for a wide range of physics and applied physics applications.

This collaborative effort would be a model for future joint national laboratory/university projects, taking advantage of the expertise and skills that both offer, to develop exciting new technologies in a timely and cost-efficient manner.

This FFAG-ERL prototypes important features of the BNL eRHIC project.

All major technical components except for the FFAG arcs already exist at Cornell University, and have mostly been commissioned. Suitable space exists at Cornell, and planning for its refurbishment has already started. 
6. References

1. E.C. Aschenauer et al., "eRHIC Design Study: An Electron-Ion Collider at BNL", arXiv:1409.1633 (2014).

2. R. Milner (ed) et al., "Proceedings of the Workshop to Explore Physics Opportunities with Intense, Polarized Electron Beams up to $300 \mathrm{MeV}$ ", Cambridge, MA, 2013 AIP Conf. Proc. 1563 , ISBN: 9780735411913.

3. D. Androi et al., "First Determination of the Weak Charge of the Proton." Physical Review Letters 111141803 (2013). doi: 10.1103/PhysRevLett.111.141803.

4. F. Albert et al., "Design of narrow-band Compton scattering sources for nuclear resonance fluorescence", Physical Review Special Topics - Accelerators and Beams 14, 050703 (2011).

5. S. Machida et al., Nature Physics 8, 243-247 (2012).

6. T. Ohkawa, University of Tokyo, Tokyo, Japan, FFAG structure suggested earlier at a Symposium on Nuclear Physics of the Physical Society of Japan in 1953 (private communication).

7. A. Kolomensky et al., Zh. Eksp. Teor. Fiz. 33, 298 (1957).

8. K. R. Symon, Phys. Rev. 100, 1247 (1955).

9. D. Trbojevic, E. D. Courant, A.A. Garren, presented at the High Energy Muon Colliders workshop in Montauk HEMC-1999, published by AIP, Woodbury, New York, USA (2000), Editor King, Bruce J. ISBN 10: 156396953X ISBN 13: 9781563969539

10. G. H. Hoffstaetter, I. Bazarov, "Beam-Breakup Instability Theory for Energy Recovery Linacs", Physical Review Special Topics - Accelerators and Beams 7, 54401 (May 2004)

11. D.R. Douglas et al., "Experimental investigation of multi-bunch, multi-pass beam breakup in the Jefferson Laboratory Free Electron Laser driver upgrade", Physical Review ST-AB 9, 064403 (2006).

12. R. Kazimi et al., "Observation and mitigation of multipass BBU in CEBAF", WEPP087, EPAC08, Genoa, Italy (2008).

13. I. Bazarov, personal communication

14. L. Cultrera et al., "Growth and characterization of rugged sodium potassium antimonide photocathodes for high brilliance photoinjector", Applied Physics Letters 103, 103504 (2013).

15. B.M. Dunham et al., "Record high-average current from a high-brightness photoinjector", Applied Physics Letters 102, 034105 (2013).

16. L. Cultrera et al., "Photocathode Behavior during High Current Running in the Cornell ERL Photoinjector", Physical Review Special Topics - Accelerators and Beams 14, 120101 (2011).

17. C. Gulliford et al., "Demonstration of low emittance in the Cornell energy recovery linac photoinjector prototype", Physical Review Special Topics - Accelerators and Beams 16, 73401 (2013).

18. G.R. Eichhorn, et al., "High Q Cavities for the Cornell ERL Main Linac", Proceedings of SRF2013, Paris, France, pp. 844-849 (2013).

19. G. Hoffstaetter et al., "The Cornell Energy Recovery Linac: Project Definition Design Report", http://www.classe.cornell.edu/Research/ERL/PDDR.html.

20. Experimental Physics and Industrial Control System, EPICS, http://www.aps.anl.gov/EPICS. 